\title{
Crecimiento y producción de enzimas lacasas de Pleurotus ostreatus durante el proceso de degradación de bisfenol a
}

\author{
Pleurotus ostreatus growth and laccase enzymes production during the degradation process \\ of bisphenol a
}

\begin{abstract}
Georgina Pérez-Montiela, José Luis Torres-Garcíaa, Libertad Juarez-Santacruzº, Diana Veronica Cortés-Espinosac, Jorge Rubio-Piña ${ }^{\mathrm{d}}$, Miriam Ahuactzin-Pérez ${ }^{\mathrm{a}}$ **

a Laboratorio de Biología Experimental, Facultad de Agrobiología, Universidad Autónoma de Tlaxcala, Autopista TlaxcalaSan Martín Km 10.5, C.P. 90120, San Felipe Ixtacuixtla, Tlaxcala, México.

b Centro de Investigación en Genética y Ambiente, Universidad Autónoma de Tlaxcala, Autopista Tlaxcala-San Martín Km 10.5, C.P. 90120, San Felipe Ixtacuixtla, Tlaxcala, México.

Centro de Investigación en Biotecnología Aplicada, Instituto Politécnico Nacional,. Carretera Estatal San Inés TecuexcomacTepetitla Km 1.5, C.P. 90700 . Tepetitla de Lardizabal, Tlaxcala, México.

d Centro de Investigación y de Estudios Avanzados del Instituto Politécnico Nacional (CINVESTAV), Departamento de Recursos del Mar, Unidad Mérida. Km. 6 Antigua Carretera a Progreso, Apdo. Postal 73-Cordemex, 97310 Mérida, Yucatán, México.
\end{abstract}

\section{RESUMEN}

El Bisfenol A [4'4'-dihidroxi-2,2 difenilpropano] (BFA) es un compuesto orgánico utilizado en una amplia gama de productos de uso doméstico. EL BFA es considerado como un potente disruptor endócrino que pone en riesgo la salud humana. En esta investigación se evaluó el crecimiento de Pleurotus ostreatus en presencia de 50 y $75 \mathrm{mg} / \mathrm{L}$ de BFA en fermentación sumergida, evaluando la; velocidad específica de crecimiento $(\mu)$, biomasa máxima $\left(X_{\max }\right)$, consumo de glucosa, porcentaje (\%) y constante de biodegradación $(k)$ del BFA, actividad enzimática de lacasas (U/L), y algunos de sus parámetros cinéticos enzimáticos. Los valores más altos con respecto a los parámetros cinéticos de crecimiento y producción de lacasas, se obtuvieron en el medio con $75 \mathrm{mg} / \mathrm{L}$ de BFA. P. ostreatus mostró valores de $\mathrm{pH}$ neuro-básicos en los medios adicionados con BFA. El consumo de glucosa fue del $100 \%$ en todos los medios. Durante las primeras $100 \mathrm{~h}$ de crecimiento, este hongo degradó más del $50 \%$ de ambas concentraciones. Esta investigación es la primera en México que demuestra la eficiencia degradante de $P$. ostreatus BE01 al degradar una concentración de BFA que no ha sido reportada anteriormente y que es superior a las reportadas en diversas matrices ambientales.

Palabras clave: Biodegradación, bisfenol A, Pleurotus ostreatus, lacasas, parámetros de crecimiento

\section{ABSTRACT}

Bisphenol A [4'4'-dihydroxy-2,2 diphenylpropane] (BPA) is an organic compound used in a wide range of household products. BPA is considered as a powerful endocrine disruptor that puts human health at risk. In this investigation, Pleurotus ostreatus growth was evaluated in presence of 50 and $75 \mathrm{mg} / \mathrm{L}$ of BPA in submerged fermentation, evaluating the specific growth rate $(\mu)$, maximum biomass $\left(X_{\max }\right)$, glucose

*Autores para correspondencia: Dra. Miriam Ahuactzin Pérez

Correo electrónico: miriamahuactzin@gmail.com

Recibido: 24 de agosto de 2020

Aceptado: 2 de noviembre de 2020 consumption, percentage (\%) and biodegradation constant (k) of BPA, enzymatic activity of laccase (U/L), and some of its enzymatic kinetic parameters. The highest values regarding the kinetic parameters of growth and laccase production were obtained in the medium with $75 \mathrm{mg} / \mathrm{L}$ of BPA. P. ostreatus showed neutral-basic $\mathrm{pH}$ values in the media added with BPA. Glucose consumption was $100 \%$ in all media. Within the first $100 \mathrm{~h}$ of growth, this fungus degraded more than $50 \%$ of both concentrations. This research is the first in México that demonstrates the degradative efficiency of $P$. ostreatus BE01 by degrading a concentration of BPA that has not been previously reported and is higher than those reported in various environmental matrices.

Keywords: Biodegradation, bisphenol A, Pleurotus ostreatus, laccases, kinetic parameters

\section{INTRODUCCIÓN}

El Bisfenol A [4'4'-dihidroxi-2,2-difenilpropano] (BFA) es un compuesto orgánico con dos grupos fenólicos, tiene una amplia gama de usos, es uno de los químicos de mayor producción a nivel mundial (Industry-Experts, 2018). En la industria este compuesto tiene una gran cantidad de aplicaciones, se utiliza como materia prima para la producción de diferentes tipos de plásticos, entre los que destacan los policarbonatos; es componente de resinas epóxicas de los recubrimientos interiores de latas de comida y se utiliza en la producción de diferentes partes de equipos eléctricos, electrónicos, automovilísticos, etc. (Mikolajewska et al., 2015). Con el paso del tiempo, el BFA es liberado al ambiente como parte de la contaminación ambiental que ocasionan los plásticos y puede ser absorbido o acumulado en organismos vivos a través de las cadenas tróficas (Wang et al., 2017; Mackay et al., 2018; Staples et al., 2018). En el caso de los seres humanos, la exposición se da vía inhalación, ingesta 
de alimentos empaquetados y a través de la dermis (Ribeiro et al., 2017; Li y Suh, 2019). Estudios recientes en roedores y humanos demostraron que el BFA afecta negativamente al sistema endocrino y con ello a la salud de estos y su descendencia, debido a su potente acción como disruptor endócrino (DE) (Adoamnei et al., 2018; Gómez-Mercado et al., 2018; Baralić et al., 2020; Gorini et al., 2020). Los DE son químicos o mezclas de ellos, exógenos al organismo que se encuentran en alimentos y productos destinados a los consumidores que muestran efectos adversos sobre el sistema endócrino (Nur et al., 2019; Yeung et al., 2019). El BFA es un compuesto aromático, químicamente resistente a la degradación, debido a la deslocalización de su energía y a las densas nubes de electrones presentes en ambos lados de la estructura de su anillo, lo que lo hace extremadamente resistente al ataque nucleofílico (Cajthaml, 2014). A pesar de esto, se han realizado diversos estudios para conocer su degradación y las enzimas que participan en dicho proceso principalmente, la participación de hongos de pudrición blanca (Baker et al., 2019). El BFA puede ser degradado por especies hongos ligninolíticos, tales como: Pleurotus ostreatus, Pleurotus eryngii, Phanerochaete chrysosporium, Trametes versicolor, Trametes villosa (Song et al., 2007; Martínková et al., 2016; Kresinová et al., 2018), Stereum hirsutum, Heterobasidium insulare y Grifola frondosa entre otros (Nitheranont et al., 2011; Traversa et al., 2012). Estos hongos excretan enzimas ligninolíticas como las lacasas y manganeso peroxidasas $(\mathrm{Mn}-\mathrm{P})$ que pueden descomponer total o parcialmente al BFA (Gassara et al., 2013; Escalona et al., 2014; Hou et al., 2014; Baker et al., 2019). Por lo que han recibido importante atención para el establecimiento de estrategias de biorremediación, debido a que catalizan la sustracción de un electrón de los grupos hidroxilo fenólicos (reacción acompañada de una desmetilación y formación de quinona) que genera la escisión del anillo (Husain y Qayyum, 2012). Una característica natural tecnológicamente útil para la degradación de diversos compuestos aromáticos. Además, el uso de hongos ligninolíticos producen mediadores de bajo peso molecular que amplían el espectro de contaminantes que son capaces de oxidar (Cajthaml, 2014; Ahuactzin-Pérez et al., 2018c). Sin embargo, existen pocos estudios que describen los parámetros cinéticos del crecimiento y producción enzimática de lacasas por $P$. ostreatus durante el proceso de degradación de BFA. Es por esto, que el propósito de este trabajo fue caracterizar el crecimiento y producción de enzimas lacasas durante el proceso de degradación de BFA por $P$. ostreatus cultivado en fermentación sumergida.

\section{MATERIALES Y MÉTODOS \\ Organismo de estudio e inóculo}

En esta investigación se utilizó el hongo P. ostreatus, proveniente de cultivos industriales, depositado y conservado en el cepario del Laboratorio de Biología Experimental con la clave BE01, de la Facultad de Agrobiología de la Universidad Autónoma de Tlaxcala. Para la obtención del inóculo, el hongo se cultivó en Agar Papa Dextrosa (PDA) durante $7 \mathrm{~d}$ a $28^{\circ} \mathrm{C}$, los inóculos se obtuvieron de la periferia de las colonias con $10 \mathrm{~mm}$ de diámetro.

\section{Medio y condiciones de cultivo}

Se utilizaron tres medios de cultivo: 1) GYE (g/L): glucosa, $10 \mathrm{~g}$; extracto de levadura, $5 \mathrm{~g} ; \mathrm{KH}_{2} \mathrm{PO}_{4^{\prime}} 0.9 \mathrm{~g} ; \mathrm{K}_{2} \mathrm{HPO}_{4^{\prime}}$ $0.6 \mathrm{~g} ; \mathrm{CuSO}_{4}-5 \mathrm{H}_{2} \mathrm{O}, 0.3 \mathrm{~g} ; \mathrm{Na}_{2} \mathrm{HPO}_{4}, 0.1 \mathrm{~g} ; \mathrm{FeSO}_{4}-7 \mathrm{H}_{2} \mathrm{O}, 0.05 \mathrm{~g}$; $\mathrm{MnSO}_{4^{\prime}} 0.05 \mathrm{~g}$ y $\left.\mathrm{ZnSO}_{4}-7 \mathrm{H}_{2} \mathrm{O}, 0.01 \mathrm{~g}\right)$. 2) $\mathrm{GYE}+50 \mathrm{mg} / \mathrm{L}$ de BFA y 3) GYE $+75 \mathrm{mg} / \mathrm{L}$ de BFA. El pH del medio se ajustó a 6.5 con $\mathrm{NaOH} 0.1 \mathrm{M}$. El hongo se cultivó en fermentación sumergida utilizando matraces Erlenmeyer de $125 \mathrm{~mL}$, a cada uno se les agregó $50 \mathrm{~mL}$ de medio de cultivo adicionado con la concentración correspondiente de BFA (50 y $75 \mathrm{mg} / \mathrm{L}$ ), se esterilizaron (a $121^{\circ} \mathrm{C}$ y 15 psi) y cada matraz se sembró con tres inóculos y fueron incubados en una incubadora orbital (PRENDO) a $120 \mathrm{rpm}$, durante 23 días a $28{ }^{\circ} \mathrm{C}$. El hongo se desarrolló, bajo una fermentación sumergida.

\section{Obtención del extracto crudo enzimático y determina- ción de la producción de biomasa}

El extracto crudo enzimático (ECE) fue el sobrenadante de los cultivos y se obtuvo por filtración (decantación) y retención de biomasa en papel filtro Whatman No. 4. La biomasa retenida sobre el papel Whatman se secó hasta peso constante para la cuantificación del crecimiento fúngico empleando el método de peso seco (Ahuactzin-Pérez et al., 2014).

Las curvas de crecimiento fúngico se establecieron mediante la ecuación logística por la minimización del error cuadrático con la herramienta Solver de la hoja electrónica de Excel (Microsoft) (Viniegra-González et al., 2003; Ahuactzin-Pérez et al., 2018c)pectinases and tannases, by Aspergillus niger grown by SSF and SmF is reviewed. To do such a comparative study, logistic and Luedeking-Piret equations are used in order to estimate the values of the following coeficients: maximal specific growth rate $(\mu \mathrm{M}$, como se muestra a continuación en la ecuación

$$
d x / d t=\mu\left(1-X / X_{\text {máx }}\right) X
$$

Donde, $\mu$ corresponde a la velocidad específica de crecimiento obtenida y $\mathrm{X}_{\text {máx }}$ a la biomasa máxima (o de equilibrio) producida cuando $d X / d t=0$ para $X>0$.

La solución de la Ec. (1) es la siguiente:

$X=X_{\text {max }} /\left(1+C^{*} \exp \left(-\mu^{*} t\right)\right.$ de la biomasa.

Donde, $C=\left(X_{\text {máx }}-X_{0}\right) / X_{0}$; cuando $X=X_{0}$ es el valor inicial

\section{Parámetros cinéticos de crecimiento y producción de enzimas lacasas}

El rendimiento de la enzima con respecto a la biomasa $\left(Y_{E X}\right)$ se estimó como la relación entre la enzima máxima producida durante la fermentación $\left(E_{\text {max }}\right)(U / L)$ y la $\left(X_{\text {max }}\right)(g / L)$. Se calculó el rendimiento de la biomasa con respecto al sustrato $\left(Y_{x y S}\right)$ empleando la $X_{\text {máx }}$ y la concentración inicial de la fuente de carbono. La productividad en el pico máximo de actividad $\left(\mathrm{P}_{\mathrm{RO}}\right)$ se calculó mediante la ecuación $\left(\mathrm{P}_{\mathrm{RO}}=E_{\text {max }} /\right.$ tiempo de fermentación y por último, la tasa específica de formación de la enzima ( $q p$ ) se determinó empleando la siguiente formula $\left(q p=\mu^{*} Y_{E X X}\right)($ Díaz et al., 2013). 


\section{Determinación del consumo de glucosa}

El consumo de glucosa se cuantificó a través de la presencia de la cantidad de azúcares reductores por el método DNS (ácido dinitrosalicílico) y una curva de calibración con glucosa (0 - $100 \mathrm{mg} / \mathrm{L}$ de glucosa). La mezcla de reacción (50 $\mu \mathrm{L}$ de $\mathrm{ECE}, 950 \mu \mathrm{L}$ de agua destilada y $2 \mathrm{~mL}$ de solución DNS $\left[\mathrm{NaOH} 1 \%, \mathrm{Na}_{2} \mathrm{SO}_{3} 0.05 \%\right.$, fenol $0.2 \%$ y DNS $\left.1 \%\right]$ ]) se calentó a punto de ebullición durante 5 min y la lectura de la absorbencia se realizó en un espectrofotómetro "METASH-UV6000" a $575 \mathrm{~nm}$ (Miller, 1959).

\section{Perfiles de $\mathrm{pH}$}

Los perfiles del pH durante el crecimiento de $P$. ostreatus en medio líquido se obtuvieron con el método de potenciometría, con el equipo Hanna instruments HI 9811-5. El potenciómetro se calibró empleando bufers de referencia a un $\mathrm{pH}$ de 4,7 y 10.

\section{Actividad enzimática de lacasas}

La actividad de lacasas se cuantificó con el sustrato 2,6-dimetoxifenol (SIGMA) $2 \mathrm{mM}$ en buffer de acetatos $0.1 \mathrm{M}$ y pH de 4.5. La mezcla de reacción $(475 \mu \mathrm{L}$ de sustrato, $50 \mu \mathrm{L}$ de ECE) se incubó a $39^{\circ} \mathrm{C}$ por 1 min. La reacción se detuvo por la adición de $475 \mu \mathrm{L}$ de dimetil sulfóxido (SIGMA). La absorbancia se leyó a $468 \mathrm{~nm}$ en un espectrofotómetro "METASHUV6000". Una unidad de actividad de lacasas es la cantidad de enzima que provoca incrementos de 1.0 unidad en la absorbancia por minuto de incubación (Díaz et al., 2013).

\section{Porcentaje y constante de biodegradación del BFA}

Para la cuantificación de la biodegradación de BFA se realizó empleando una alícuota de $1 \mathrm{~mL}$ de ECE por cada réplica de la fermentación (3) y se sometió a shock térmico (a temperatura de ebullición) durante 1 minuto, se evaporó en un horno de secado a $50^{\circ} \mathrm{C}$ durante $24 \mathrm{~h}$. Posteriormente, para su cuantificación y análisis la alícuota se resuspendió en $2 \mathrm{~mL}$ de acetonitrilo. Los extractos se analizaron con un sistema HPLC Agilent 110 de fase inversa, equipado con una columna C18 y un detector de absorbencia UV de 245-360 $\mathrm{nm}$ en condiciones isocráticas en acetonitrilo:agua (90:10) y a un caudal de $1 \mathrm{~mL} / \mathrm{min}$.

La biodegradación de BFA se determinó midiendo su desaparición en el medio de cultivo. Se realizó una curva de calibración, en un intervalo de concentraciones de 0-100 ppm de BFA con el comportamiento de la Ec (2).

$$
\mathrm{Y}=\mathrm{mx}+\mathrm{b}
$$

Donde, $x$ es la concentración correspondiente a cada área bajo la curva de los días de fermentación. El porcentaje de biodegradación (\% BD) en el intervalo de tiempo (h) se calculó mediante la

$$
\% B D t=\frac{C t}{C o} \times 10
$$

Donde:

$C_{0}=$ concentración inicial del BFA en la solución de ensayo $(\mathrm{mg} / \mathrm{L})$.

$C_{t}=$ concentración de BFA en la solución de ensayo en el tiempo $t$.

Se graficó el tiempo de fermentación en comparación con el logaritmo natural (In) de la concentración de BFA, posteriormente se aplicó una regresión lineal. La pendiente de la regresión se consideró la constante de biodegradación de primer orden ( $k$ ) expresada en $\mathrm{h}^{-1}$ (Ahuactzin-Pérez et al., 2016; Ahuactzin-Pérez et al., 2018c).

\section{Análisis estadístico}

Todos los experimentos se llevaron a cabo por triplicado, empleando un diseño experimental unifactorial completamente aleatorizado de tres niveles. Los datos se evaluaron mediante un ANOVA de una vía y una prueba post-hoc de Tukey con el programa The Graph Pad Prism ${ }^{\oplus}$ program (San Diego, CA, USA) (Córdoba-Sosa et al., 2014).

\section{RESULTADOS}

\section{Parámetros cinéticos de crecimiento}

En el medio GYE se obtuvo un valor de $3.45 \mathrm{~g} / \mathrm{L}$ para la $X_{\text {máx }}$ el cual fue menor con respecto a los medios de cultivo con BFA. En el mismo medio se obtuvo un valor menor de $\mu$ con respecto a los tratamientos con BFA (Fig. 1). Sin embargo, el valor de $X_{\text {max }}$ no fue significativamente diferente de los otros tratamientos. El hongo $P$. ostreatus presentó un valor de $X_{\text {máx }}$ de $4.9 \mathrm{~g} / \mathrm{L}$ en el medio que contenía $50 \mathrm{mg} / \mathrm{L}$ de BFA y una $\mu$ de $0.01 \mathrm{~h}^{-1}$. En comparación con el medio con $75 \mathrm{mg} / \mathrm{L}$ de BFA en donde se presentó una $X_{\text {máx }}$ de $5.3 \mathrm{~g} / L$ y una $\mu 0.014$ $\mathrm{h}^{-1}$ (Tabla 1, Fig. 1). No hubieron diferencias significativas entre las medias de los valores correspondientes a la $X_{\text {máx }}$ y a la $\mu$ en todos los tratamientos $(p<0.05)$.

\section{Consumo de glucosa}

El hongo consumió el $100 \%$ de la glucosa en el tratamiento testigo y en el medio con $75 \mathrm{mg} / \mathrm{L}$ de BFA después de $432 \mathrm{~h}$ de crecimiento (Fig. 2). Mientras que en el medio con $50 \mathrm{mg} / \mathrm{L}$ de BFA el consumo total de glucosa fue lento (transcurridas $480 \mathrm{~h}$ de crecimiento) (Fig. 2). En todos los

Tabla 1. Parámetros cinéticos de crecimiento de $P$. ostreatus cultivado sobre BFA en fermentación sumergida

Table 1. Kinetic growth parameters of $P$. ostreatus grown on BPA in submerged fermentation

\begin{tabular}{lcccc}
\hline \multicolumn{5}{c}{ Medio de cultivo } \\
Parámetro & \multicolumn{4}{c}{ BFA (mg/L) } \\
\cline { 2 - 5 } & $\mathbf{0}$ & $\mathbf{5 0}$ & $\mathbf{7 5}$ & $\begin{array}{c}\text { Valor } \\
\text { de } \mathbf{P}\end{array}$ \\
\hline$\mu\left(\mathrm{h}^{-1}\right)$ & $0.008 \pm 0.0005^{\mathrm{c}}$ & $0.010 \pm 0.0009^{\mathrm{b}}$ & $0.014 \pm 0.0003^{\mathrm{a}}$ & 0.0051 \\
$\mathrm{X}_{\text {máx }}(\mathrm{g} / \mathrm{L})$ & $3.45 \pm 0.104^{\mathrm{c}}$ & $4.9 \pm 0.04^{\mathrm{b}}$ & $5.3 \pm 0.01^{\mathrm{a}}$ & 0.0001 \\
$\mathrm{Y}_{(\mathrm{X} / \mathrm{s})}\left(\mathrm{g}_{\mathrm{x}} / \mathrm{g}_{\mathrm{s}}\right)$ & $0.34 \pm 0.01^{\circ} \mathrm{C}$ & $0.49 \pm 0.004^{\mathrm{b}}$ & $0.52 \pm 0.001^{\mathrm{a}}$ & 0.0002 \\
\hline
\end{tabular}

$\mathrm{X} \pm$ d.s. $\mathrm{a}, \mathrm{b}, \mathrm{c}$ Literales diferentes en la misma fila indica diferencias significativas $(p<0.05)$. 


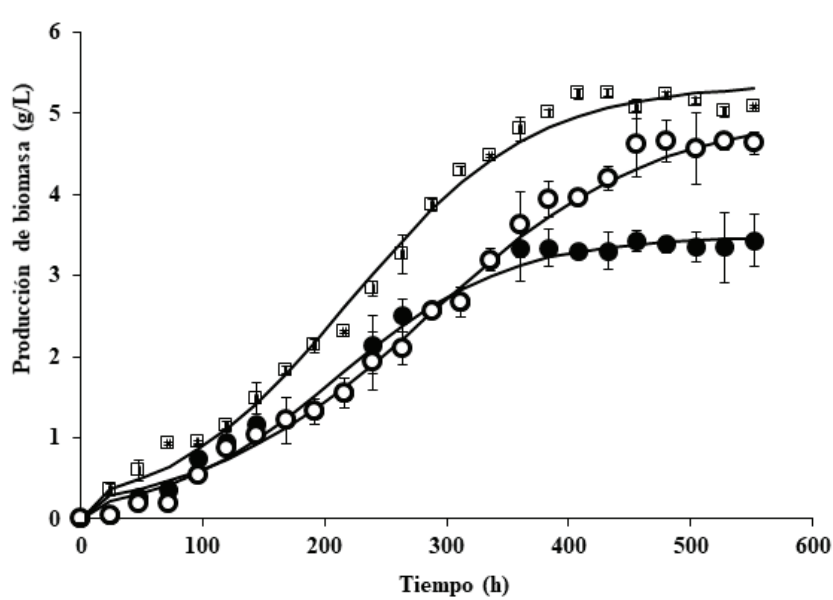

Figura 1. Crecimiento y producción de biomasa de $P$. ostreatus en medio

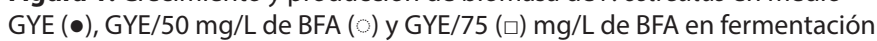
sumergida, determinado por el método de peso seco.

Figure 1. Growth and biomass production of $P$. ostreatus in GYE $(\bullet), G Y E / 50$ $\mathrm{mg} / \mathrm{L}$ of BPA (।) and GYE/75 ( $\square$ ) $\mathrm{mg} / \mathrm{L}$ of BPA medium in submerged fermentation, determined by the dry weight method.

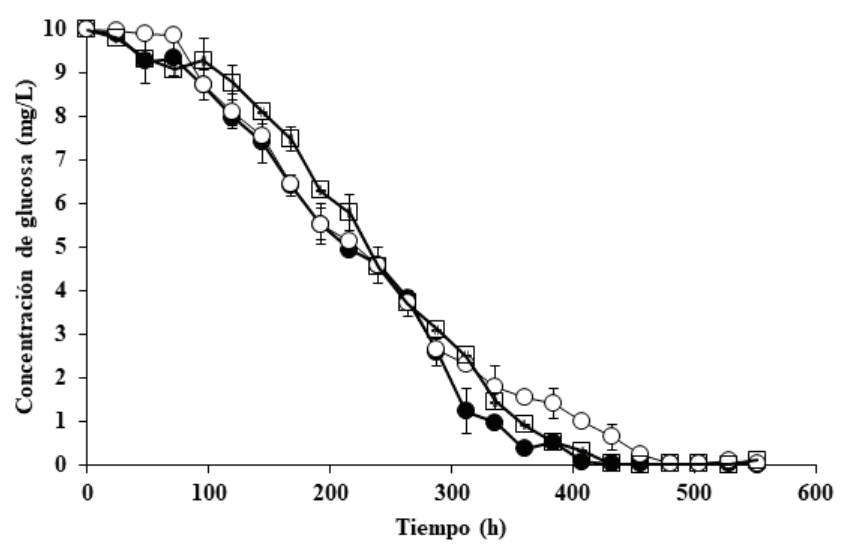

Figura 2. Consumo de glucosa por P. ostreatus en medio GYE (•), GYE/50 $\mathrm{mg} / \mathrm{L}$ de BFA () y GYE/75 ( $\square$ ) mg/L de BFA en fermentación sumergida, determinado por el método de DNS.

Figure 2. Glucose consumption by P. ostreatus in GYE (•), GYE/50 mg/L of BFA ( ) and GYE/75 ( $\square$ ) $\mathrm{mg} / \mathrm{L}$ of BPA medium in submerged fermentation, determined by the DNS method.

tratamientos, el consumo de glucosa total correspondió con el inicio de la fase estacionaria de crecimiento de $P$. ostreatus

\section{Perfiles de pH}

En el perfil de $\mathrm{pH}$ durante el crecimiento de P. ostreatus en el medio GYE se observó una disminución progresiva de 6.2 a 4.7 entre las 48 y 192 h (fase de adaptación y exponencial), respectivamente. Posteriormente, un aumento del $\mathrm{pH}$ hasta 7.4 a las $552 \mathrm{~h}$ se registró. En el medio con $50 \mathrm{mg} / \mathrm{L}$ de BFA se observó un intervalo de $\mathrm{pH}$ de 5.8 a 4.2 durante las primeras $336 \mathrm{~h}$ de crecimiento fúngico, hasta llegar a un $\mathrm{pH}$ de 6.5 al final de la fermentación. El perfil de $\mathrm{pH}$ en el medio con $75 \mathrm{mg} / \mathrm{L}$ de BFA mostró una disminución durante las primeras 96 h. Después, el pH alcanzó un valor de 6.3 que incrementó al finalizar la fermentación a 7.5 (Fig. 3).

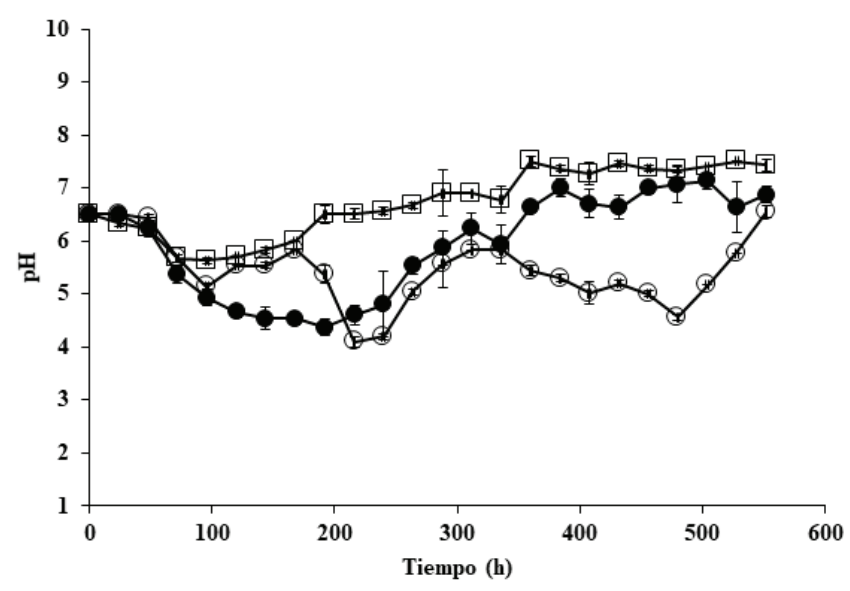

Figura 3. Perfiles de $\mathrm{pH}$ de $P$. ostreatus cultivado en fermentación sumergida con diferentes concentraciones de BFA: $0(\bullet), 50$ (o) y 75 ( $\square$ ) mg/L.

Figure 3. $\mathrm{pH}$ profiles of $P$. ostreatus grown in submerged fermentation with different BPA concentrations: 0 (•), 50 (o) and 75 (口) mg/L.

\section{Actividad enzimática de lacasas}

La mayor actividad de enzimas lacasas se presentó en el medio con mayor concentración de BFA, con un valor de $238 \mathrm{U} / \mathrm{L}$ a las $336 \mathrm{~h}$ (Tabla 2, Fig. 4). Con respecto al medio con $50 \mathrm{mg} / \mathrm{L}$ de BFA, la producción de estas enzimas durante las primeras horas de crecimiento fue menor a $10 \mathrm{U} / \mathrm{L}$. Sin embargo, las $159 \mathrm{U} / \mathrm{L}$ se alcanzaron a las $288 \mathrm{~h}$ (Fig. 4). La menor actividad enzimática se mostró en el medio GYE con un máximo de $33 \mathrm{U} / \mathrm{L}$ a las $192 \mathrm{~h}$ de crecimiento (Fig. 4).

\section{Parámetros cinéticos de producción de lacasas}

Con respecto a los parámetros de $E_{\text {máx' }} Y_{E / X_{1}} P_{R O}$ y $q_{p^{\prime}}$ se presentaron los valores mayores en el medio $75 \mathrm{mg} / \mathrm{L}$ de BFA. Valores significativamente diferentes de los obtenidos en el medio GYE $(p<0.05)$ (Tabla 2). El valor de $Y_{E / X}$ para el medio GYE no presentó diferencia significativa $(p<0.05)$ con respecto a los medios que contienen BFA. Sin embargo, en el medio con $50 \mathrm{mg} / \mathrm{L}$ de BFA, el valor de $Y_{E / X}$ fue significativamente diferente al que presentó el medio de crecimiento con la mayor concentración de BFA, mientras que el medio GYE fue

Tabla 2. Parámetros cinéticos de producción de lacasas de $P$. ostreatus cultivado sobre BFA en fermentación sumergida.

Table 2. Laccase production kinetic parameters of $P$. ostreatus grown on BPA in submerged fermentation.

\begin{tabular}{|c|c|c|c|c|}
\hline \multirow{3}{*}{ Parámetro } & \multicolumn{3}{|c|}{ Medio de cultivo } & \multirow[b]{3}{*}{$\begin{array}{l}\text { Valor } \\
\text { de P }\end{array}$} \\
\hline & \multicolumn{3}{|c|}{ BFA (mg/L) } & \\
\hline & 0 & 50 & 75 & \\
\hline $\mathrm{E}_{\text {máx }}(\mathrm{U} / \mathrm{L})$ & $33.17 \pm 15.3^{4} \mathrm{C}$ & $210 \pm 10.8^{3} \mathrm{~b}$ & $238.24 \pm 1.2^{8} \mathrm{a}$ & 0.0008 \\
\hline$Y_{E / X}(U / g X)$ & $25 \pm 11.56^{c}$ & $65.93 \pm 3.39^{a}$ & $53.43 \pm 0.29^{b}$ & 0.0051 \\
\hline$P_{R O}(U L / h)$ & $0.17 \pm 0.08^{c}$ & $0.63 \pm 0.03^{a}$ & $0.71 \pm 0.003^{b}$ & 0.0002 \\
\hline
\end{tabular}

$X \pm$ d.s. $a, b$, .c Literales diferentes en la misma fila indica diferencias significativas $(p<0.05)$. 


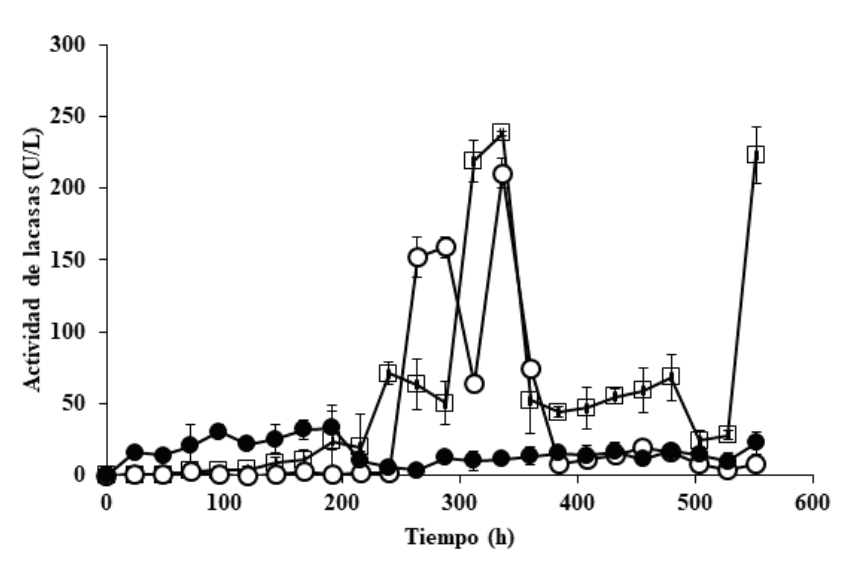

Figura 4. Actividad de lacasas de $P$. ostreatus en medio GYE (•), GYE/50 $\mathrm{mg} / \mathrm{L}$ de BFA ( ) y GYE /75 ( $\square$ ) mg/L de BFA en fermentación sumergida. Figure 4. Laccase activity of $P$. ostreatus in GYE (•), GYE/50 mg/L of BPA (०) and GYE/75 (口) $\mathrm{mg} / \mathrm{L}$ of BPA medium in submerged fermentation.

el que mostró menor rendimiento (Tabla 2). Los valores de la $E_{\text {máx }} P_{R O}$ y $q_{p}$ mostraron diferencias significativas $(\boldsymbol{p}<0.05)$ en el medio GYE y en los medios con BFA, estos parámetros no mostraron diferencia significativa (Tabla 2).

\section{Porcentaje y constante de biodegradación del BFA}

En el medio con $75 \mathrm{mg} / \mathrm{L}$ de BFA, después de $552 \mathrm{~h}$ de crecimiento en fermentación líquida, $P$. ostreatus degradó hasta un $94.3 \%$ de dicho compuesto. Durante el inicio de la fase de adaptación (las primeras $48 \mathrm{~h}$ ) el hongo degradó el $67.7 \%$ de la concentración inicial (Fig. 6a). Para este medio se obtuvo un valor de $k$ de $0.0045 \mathrm{~h}^{-1}$ (Fig. 6b). En el medio con menor concentración de BFA, P. ostreatus degradó más del 50 $\%$ de la concentración inicial $(50 \mathrm{mg} / \mathrm{L})$ después de $96 \mathrm{~h}$ de incubación y alcanzó un $98.3 \%$ de degradación a las 552 h, y un valor de $k$ de $0.0051 \mathrm{~h}^{-1}$ (Fig. 5a,b).

\section{DISCUSIÓN}

En esta investigación se utilizó el modelo de la "ecuación logística" para explicar el crecimiento de $P$. ostreatus en

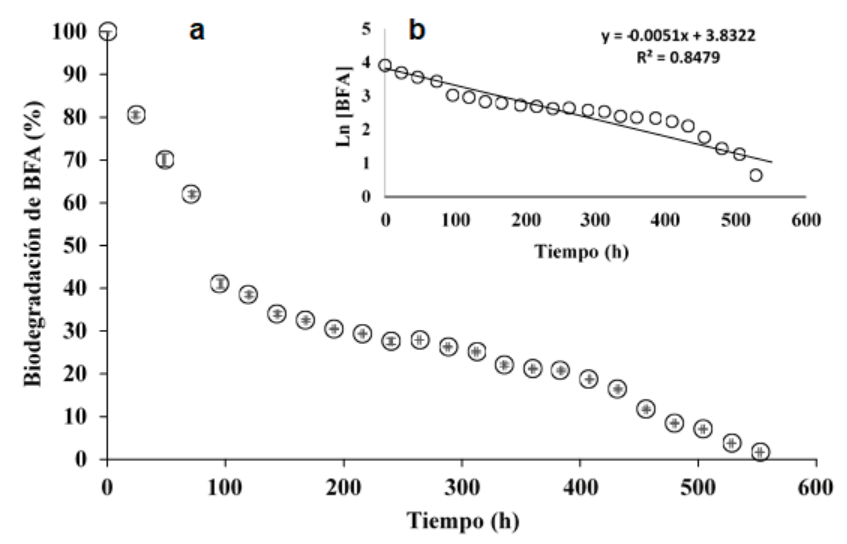

Figura 5. Porcentaje de biodegradación de $50 \mathrm{mg} / \mathrm{L}$ de BFA (a) y constante de biodegradación (b).

Figure 5. Biodegradation percentage of $50 \mathrm{mg} / \mathrm{L} \mathrm{BFA} \mathrm{(a)} \mathrm{and} \mathrm{biodegrada-}$ tion constant (b).

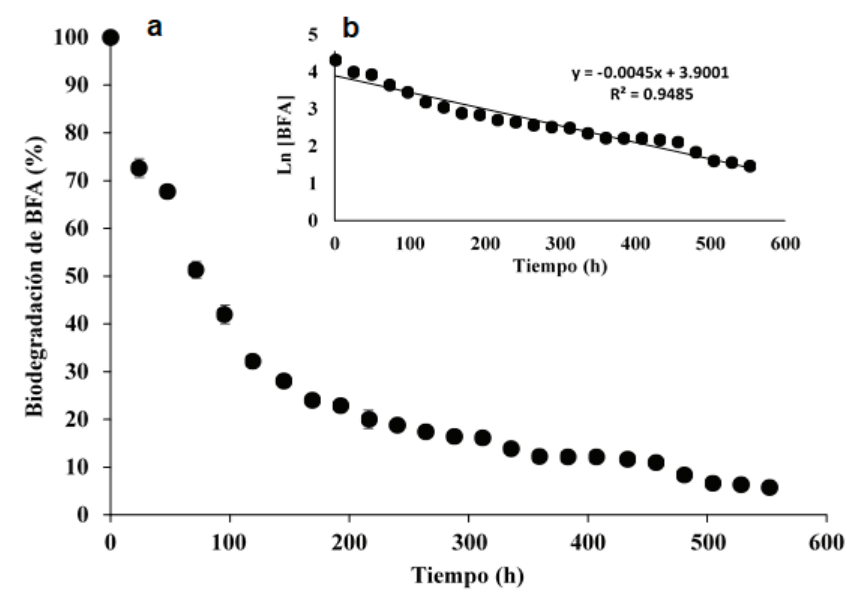

Figura 6. Porcentaje de biodegradación de $75 \mathrm{mg} / \mathrm{L}$ de BFA (a) y constante de biodegradación (b).

Figure 6. Biodegradation percentage of $75 \mathrm{mg} / \mathrm{L}$ BFA (a) and biodegradation constant (b).

fermentación sumergida. El criterio de selección del modelo fue que en las cinéticas de crecimiento fúngico mostró un coeficiente de correlación mayor a 99 \%. Este modelo describió el comportamiento del crecimiento de este hongo bajo las condiciones evaluadas de fermentación. Ahuactzin-Pérez et al. (2018a) evaluaron la degradación de 500 y 1000 mg/L de di (2-etilhexil) ftalato (DEHF) (un compuesto fenólico plastificante en la industria de plásticos) por una cepa diferente de $P$. ostreatus. Ellos reportaron en tratamientos testigo y con concentraciones de DEHF la obtención de una $X_{\text {máx }}$ de $5.19,6.53$ y $7.69 \mathrm{~g} / \mathrm{L}$, respectivamente. Así como, una $\mu$ de $0.21,0.022$ y $0.018 \mathrm{~h}^{-1}$. Estos resultados son similares a los descritos en este trabajo. En la observación de que en los medios de cultivo adicionados con la mayor concentración de BFA se obtuvieron los mayores rendimientos $(3.45,4.9 \mathrm{y}$ $5.3 \mathrm{~g} / \mathrm{L}$ en los medios GYE, 50 y $75 \mathrm{mg} / \mathrm{L}$ de BFA) de $X_{\text {máx' }}$, pero una menor $\mu$ (0.0088, 0.01 y $0.014 \mathrm{~h}^{-1}$ respectivamente). Sin embargo, estos resultados difieren con los obtenidos por Tlecuitl-Beristain et al. (2008), quienes reportan para esta especie después de $480 \mathrm{~h}$ de crecimiento en medio líquido sin la adición de algún xenobiótico; una $\mu$ y $X_{\text {máx }}$ superior de 0.02 $\mathrm{h}^{-1}$ y $7.8 \mathrm{~g} / \mathrm{L}$, respectivamente. Ahuactzin-Pérez et al. (2016) reportaron el consumo total de glucosa por el hongo Fusarium culmorum después de 290 y $240 \mathrm{~h}$ de crecimiento sobre 500 y 1000 mg/L de DEHF, respectivamente. En comparación con los datos obtenidos en esta investigación, el consumo total de glucosa en el medio GYE y con $75 \mathrm{mg} / \mathrm{L}$ de BFA por $P$. ostreatus se observó a las $432 \mathrm{~h}$. Sin embargo, en el medio con $50 \mathrm{mg} / \mathrm{L}$ de BFA se prolongó hasta las $480 \mathrm{~h}$.

En cuanto al efecto del $\mathrm{pH}$ en el crecimiento fúngico, en los tres medios se muestran cambios similares a los reportados por Ahuactzin-Pérez et al. (2016). Mediante el ajuste del $\mathrm{pH}$ inicial a 6.5 en los tres tratamientos (GYE, 500 y $1000 \mathrm{mg} / \mathrm{L}$ de DEHF) se observó un pH final de $6.8,6.4$ y 4.5, respectivamente. En el presente trabajo se obtuvo un $\mathrm{pH}$ final de 6.8, 6.4 y 7.4 para los tratamientos testigo, con 50 y $75 \mathrm{mg} / \mathrm{L}$ de BFA, respectivamente. Con respecto a la actividad enzimática 
de lacasas, Ortega-Ávila (2015) describió el comportamiento de la producción de lacasas en el medio GYE adicionado con $\mathrm{Cu}$ como inductor, donde obtuvo una $E_{\max }$ de 120 y 119 $\mathrm{U} / \mathrm{L}$ a las 312 y $336 \mathrm{~h}$ de crecimiento, respectivamente. La comparación de los resultados obtenidos en este trabajo, la actividad de lacasas fue menor a la reportado por OrtegaÁvila (2015). Sin embargo, los resultados indican que el BFA o los intermediarios de su degradación ejercen un efecto inductor sobre la producción de estas enzimas. Si bien, en esta investigación no se evaluó el efecto inductivo del BFA sobre los perfiles de enzimas lacasas, se conoce que estas enzimas son responsables de la degradación de este compuesto (Hou et al., 2014; Neiverth de Freitas et al., 2017; Kresinová et al., 2018). Díaz et al. (2013) mencionan que la mayor producción de enzimas lacasas para $P$. ostreatus se presenta con un $\mathrm{pH}$ inicial de crecimiento de 6.5 en el medio de cultivo y a un $\mathrm{pH}$ de actividad de 4.5, en medios sin presencia de BFA. Este comportamiento se observó en el medio testigo empleado en nuestra investigación, contrario a los medios adicionados con BFA, donde la actividad y parámetros cinéticos enzimáticos fueron mayores, por lo que nos permite sugerir que estos incrementos se deben a la presencia de BFA y no al pH.

Díaz et al. (2011) evaluaron diferentes parámetros cinéticos de producción $\left(E_{\text {max́ }} Y_{E X^{\prime}} P\right.$ y la $q_{p}$ ) en diversas cepas del género Pleurotus cultivado en medios de cultivo, con y sin la adicción de $\mathrm{Cu}^{++}$. La observación fue que la cepa de $P$. ostreatus ATCC 32783 mostró valores mayores en los parámetros cinéticos de producción de la enzima lacasa en presencia de $\mathrm{Cu}^{++}$en el medio de cultivo. En esta investigación se observó que $P$. ostreatus cultivado en los medios conteniendo BFA, obtuvo valores de los parámetros cinéticos de producción de la enzima menores a los obtenidos por Díaz et al. (2011).

Los hongos T. versicolor, P. ostreatus, Irpex lacteus y $S$. hirsutum han sido especies de hongos ligninolíticos que han degradado por arriba del $95 \%$ de la concentración inicial (50 $\mathrm{mg} / \mathrm{L}$ ) en un lapso de 5 a 7 días crecidas en medios líquidos y sólidos en un rango de $\mathrm{pH}(4.5-5)$ y temperatura de $\left(20-30^{\circ} \mathrm{C}\right)$ (Song et al., 2007; Traversa et al., 2012). Por otro lado, Cajthaml et al. (2009) y Zhang et al. (2015) reportan la degradación de $10 \mathrm{mg} / \mathrm{L}$ de BFA por cepas de P. ostreatus. Los resultados mostraron una eficiencia de degradación del $99 \%$ de la concentración inicial a los 3 y 7 días del crecimiento de $P$. ostreatus, respectivamente. Contrario a lo reportado por estos autores, en esta investigación se emplearon concentraciones 5 y 7.5 veces mayores a las reportadas por ellos. P. ostreatus degradó más del $50 \%$ de la concentración inicial a partir de los primero 4 y 3 días, a una $k$ de 0.0045 y $0.0051 \mathrm{~h}^{-1}$, respectivamente. Estos resultados sugieren que $P$. ostreatus logra degradar concentraciones superiores a las que se encuentran en diferentes muestras de agua que ponen en riesgo la salud pública (Nur et al., 2019; Petrie et al., 2019; Zhang et al., 2019).

\section{CONCLUSIONES}

Durante el proceso de degradación de BFA en un sistema de fermentación sumergida, $P$. ostreatus logró incrementar sus parámetros cinéticos de crecimiento y producción de enzimas lacasas, parámetros, inversamente proporcionales a la degradación de BFA. P. ostreatus puede utilizar al BFA como fuente secundaria de carbono durante un cometabolismo, esto con base en el consumo total de la glucosa. P. ostreatus mostró valores de pH ácidos en el medio testigo e incrementaron en un rango neutro-básico en los medios que contenían BFA, lo que nos permitió inferir que el incremento en los valores de este parámetro es una medida indirecta de la degradación de este compuesto. Se sugiere que el BFA y algunos de sus subproductos podrían tener un efecto inductor en la producción de lacasas, mostrando una correlación positiva entre la concentración de BFA y la producción de estas enzimas. Esta cepa fúngica es eficiente en la biodegradación de BFA, ya que presentó un porcentaje de degradación mayor al $50 \%$ durante los primeros $3 \mathrm{~d}$ de crecimiento y finalizó a los 21 d con más del $97 \%$ de la concentración inicial más alta, concentración que hasta el momento no ha sido reportado por otros autores y que es superior a las reportadas en matrices ambientales que ponen en riesgo la salud humana.

\section{REFERENCIAS}

Adoamnei, E., Mendiola, J., Vela-Soria, F., Fernández, M.F., Olea, N., Jørgensen, N., Swan, S.H. y Torres-Cantero, A.M. 2018. Urinary bisphenol A concentrations are associated with reproductive parameters in young men. Environmental Research. 161: 122-128.

Ahuactzin-Pérez, M., Tlecuitl-Beristain, S., García-Dávila, J., González-Pérez, M., Gutiérrez-Ruíz, M.C. y Sánchez, C. 2016. Degradation of di (2-ethyl hexyl) phthalate by Fusarium culmorum: Kinetics, enzymatic activities and biodegradation pathway based on quantum chemical modeling pathway based on quantum chemical modeling. Science of the Total Environment. 566-567: 1186-1193.

Ahuactzin-Pérez, M., Tlecuitl-Beristain, S., García-Dávila, J., Santacruz-Juárez, E., González-Pérez, M., Gutiérrez-Ruíz, M.C. y Sánchez, C. 2018a. Mineralization of high concentrations of the endocrine disruptor dibutyl phthalate by Fusarium culmorum. 3 Biotech. 8: 42.

Ahuactzin-Pérez, M., Tlécuitl-Beristain, S., García-Dávila, J., Santacruz-Juárez, E., González-Pérez, M., Gutiérrez-Ruíz, M.C. y Sánchez, C. 2018c. Kinetics and pathway of biodegradation of dibutyl phthalate by Pleurotus ostreatus. Fungal Biology. 122: 991-997.

Ahuactzin-Pérez, M., Torres, J.L., Rodríguez-Pastrana, B.R., Soriano-Santos, J., Díaz-Godínez, G., Díaz, R., TlecuitlBeristain, S. y Sánchez, C. 2014. Fungal biodegradation of dibutyl phthalate and toxicity of its breakdown products on the basis of fungal and bacterial growth. World Journal of Microbiology and Biotechnology. 30: 2811-2819.

Baker, P., Tiroumalechetty, A. y Mohan, R. 2019. Fungal enzymes for bioremediation of xenobiotic compounds, in:Yadav, A.N., Singh, S., Mishra, S. y Gupta, A. (Eds.), Recent advancement in white biotechnology through fungi. pp 463-490. Springer, Cham.

Baralić, K., Djordjevic, A.B., Živančević, K., Antonijević, E., Andelkovic, M., Jovarac, D., Curcic, M., Bulat, Z., Antonijević, B. y Dukic-Cosic, D. 2020. Toxic effects of the mixture of phthalates and bisphenol A-subacute oral toxicity study in wistar rats. International Journal of Environmental Research Public Health. 17: 746. 
Cajthaml, T. 2014. Biodegradation of endocrine-disrupting compounds by ligninolytic fungi: Mechanisms involved in the degradation. Environmental Microbiology. 17: 48224834.

Cajthaml, T., Kresinová, Z., Svobodová, K. y Möder, M. 2009. Biodegradation of endocrine-disrupting compounds and suppression of estrogenic activity by ligninolytic fungi. Chemosphere. 75: 745-750.

Córdoba-Sosa, G., Torres, J.L., Ahuactzin-Pérez, M., Godínez, G., Díaz, R. y Sánchez, C. 2014. Growth of Pleurotus ostreatus ATCC 3526 in different concentrations of di (2-ethylhexyl) phthalate in submerged fermentation. Journal Chemical, Biology and Physical Science. 4: 96-103.

Díaz, R., Alonso, S., Sánchez, C., Tomasini, A., Bibbins-Martínez, M. y Díaz-Godínez, G. 2011. Characterization of the growth and laccase activity of strains of Pleurotus ostreatus in submerged fermentation. BioResources. 6: 282-290.

Díaz, R., Téllez-Téllez, M., Sánchez, C., Bibbins-Martínez, M.D., Díaz-Godínez, G. y Soriano-Santos, J. 2013. Influence of initial $\mathrm{pH}$ of the growing medium on the activity, production and genes expression profiles of laccase of Pleurotus ostreatus in submerged fermentations. Electronic Journal of Biotechnology. 16.

Escalona, I., Grooth, J. De, Font, J. y Nijmeijer, K. 2014. Removal of BPA by enzyme polymerization using NF membranes. Journal of Membrane Science. 468: 192-201.

Gassara, F., Brar, S.K., Verma, M. y Tyagi, R.D. 2013. Bisphenol A degradation in water by ligninolytic enzymes. Chemosphere. 92(10): 1356-1360.

Gorini, F., Bustaffa, E., Coi, A., lervasi, G. y Bianchi, F. 2020. Bisphenols as environmental triggers of thyroid dysfunction: Clues and evidence. International Journal of Environmental Research Public Health. 17(8): 2654.

Hou, J., Dong, G., Ye, Y. y Chen, V. 2014. Enzymatic degradation of bisphenol-A with immobilized laccase on $\mathrm{TiO}_{2}$ sol-gel coated PVDF membrane. Journal of Membrane Science. 469: 19-30.

Husain, Q. y Qayyum, S. 2012. Biological and enzymatic treatment of bisphenol $A$ and other endocrine disrupting compounds: A review. Critical Reviews in Biotechnology. 33: 260-292.

Industry-Experts. Bisphenol A - Global Market Outlook (20172026). [Consultado 12 Julio 2020] 2018. Disponible en: https://www.researchandmarkets.com/reports/4613546/ bisphenol-a-global-market-outlook-2017-2026.

Gómez-Mercado, C.A., Mejía-Sandoval, G., Segura-Cardona, A.M., Arango-Alzate, C.M., Hernández-González, S.I., Patiño-García, D.F. y Barraza-Villarreal, A. 2018. Exposición a bisfenol a (BFA) en mujeres embarazadas y su relación con la obesidad en sus hijos: revisión sistemática. Revista Facultad Nacional de Salud Pública. 36(1): 66-74.

Kresinová, Z., Linhartová, L., Filipová, A., Ezechiáš, M., Mašín, P. y Cajthaml, T. 2018. Biodegradation of endocrine disruptors in urban wastewater using Pleurotus ostreatus bioreactor. New Biotechnology. 43: 53-61.

Li, D. y Suh, S. 2019. Health risks of chemicals in consumer products: A review. Environment International. 123:580-587.

Mackay, D., Celsie, A.K.D., Powell, E. y Parnis, J.M.P. 2018. Bioconcentration, bioaccumulation, biomagnification and trophic magnification: a modelling perspective. Environmental Science: Processes and Impacts. 20: 72-85.
Martínková, L., Kotik, M., Marková, E. y Homolka, L. 2016. Biodegradation of phenolic compounds by Basidiomycota and its phenol oxidases: A review. Chemosphere. 149: 373382.

Mikolajewska, K., Stragierowicz, J. y Gromadzinska, J. 2015. Bosphenol A-applications, sources of exposure and potential risks in infants, children and pregnant women. International Journal of Occupational Medicine and Environmental Health. 28: 209-241.

Miller, G.L. 1959. Use of dinitrosalicylic acid reagent for determination of reducing sugar. Analytical Chemestry. 31: 426-428.

Neiverth de Freitas, E., Bubna, G.A., Brugnari, T., Kato, C.G., Nolli, M., Rauen, T.G., Peralta-Muniz Moreira, R. de F. y Peralta, R.A. 2017. Removal of bisphenol A by laccases from Pleurotus ostreatus and Pleurotus pulmonarius and evaluation of ecotoxicity of degradation products. Chemical Engineering Journal. 330: 1361-1369.

Nitheranont, T., Watanabe, A., Suzuki, T., Katayama, T. y Asada, Y. 2011. Decolorization of synthetic dyes and diodegradation of bisphenol A by laccase from the edible mushroom, Grifola frondosa. Bioscience, Biotechnology and Biochemistry. 75: 1845-1847.

Nur, A., Yee, S., Hirawaty, N. y Zaharin, A. 2019. Quantification of multi-classes of endocrine-disrupting compounds in estuarine wáter. Envirionmental Pollution. 249: 1019-1028.

Ortega-Ávila, R. 2015. Búsqueda de los genes que codifican para lacasas e isoenzimas producidas por la cepa PoB de Pleurotus ostreatus en dos condiciones de cultivo sobre agar. Tesis de Licenciatura. Universidad Autónoma de Tlaxcala. Tlaxcala, México.

Petrie, B., Lopardo, L., Proctor, K., Youdan, J., Barden, R. y Kasprzyk-Hordern, B. 2019. Assessment of bisphenol-A in the urban water cycle. Science of the Total Environment. 650: 900-907.

Ribeiro, E., Ladeira, C. y Viegas, S. 2017. Occupational exposure to bisphenol A (BPA): A reality that still needs to be unveiled. Toxics. 5: 1-16.

Song, H.H., Hyoung, T.C. y Hong-Gyu, S. 2007. Biodegradation of endocrine-disrupting bisphenol $A$ by white rot fungus. Journal Microbiology and Biotechnology. 17: 1147-1151.

Staples, C., Hoeven, N. Van Der, C.K., Mihaich, E., Woelz, J. y Hentges, S. 2018. Distributions of concentrations of bisphenol A in North American and European surface waters and sediments determined from 19 years of monitoring data. Chemosphere. 201: 448-458.

Tlecuitl-Beristain, S., Sánchez, C., Loera, O., Robson, G.D. y DíazGodínez, G. 2008. Laccases of Pleurotus ostreatus observed at different phases of its growth in submerged fermentation: production of a novel laccase isoform. Mycological Research. 112: 1080-1084.

Traversa, A., Loffredo, E., Gattullo, C.E. y Senesi, N. 2012. Biodecontamination of aqueous substrates from bisphenol A by ligninolytic fungi. Journal of Environmental Science and Health, Part A. 4529: 1407-1412.

Viniegra-González, G., Favela-Torres, E., Aguilar, C.N., RómeroGomez, S. de J., Díaz-Godínez, G. y Augur, C. 2003. Advantages of fungal enzyme production in solid state over liquid fermentation systems. Biochemical Engineering Journal. 13: 157-167. 
Wang, Q., Chen, M., Shan, G., Chen, P., Cui, S., Yi, S. y Zhu, L. 2017. Bioaccumulation and biomagni fi cation of emerging bisphenol analogues in aquatic organisms from Taihu Lake, China. Science of the Total Environment. 598: 814-820.

Yeung, E.H., Bell, E.M., Sundaram, R., Ghassabian, A., Ma, W., Kannan, K. y Louis, G.M. 2019. Examining endocrine disruptors measured in newborn dried blood spots and early childhood growth in a prospective cohort. Obesity. 27(1): 145-151.
Zhang, C., Li, M., Chen, X. y Li, M. 2015. Edible fungus degrade bisphenol A with no harmful effect on its fatty acid composition. Ecotoxicology and Environmental Safety. 118: 126-132.

Zhang, H., Zhang, Y., Li, J. y Yang, M. 2019. Occurrence and exposure assessment of bisphenol analogues in source water and drinking water in China. Science of the Total Environment. 655: 607-613. 